\title{
First-Passage Exponent in Two-Dimensional Soap Froth
}

\author{
W. Y. Tam, ${ }^{1}$ R. Zeitak, ${ }^{2}$ K. Y. Szeto, ${ }^{1}$ and J. Stavans ${ }^{1,3}$ \\ ${ }^{1}$ Department of Physics, The Hong Kong University of Science and Technology, Clear Water Bay, Kowloon, Hong Kong \\ ${ }^{2}$ Laboratoire de Physique Statistique, Ecole Normale Superieure, 24 Rue Lhomond, 75231 Paris Cedex 05, France \\ ${ }^{3}$ Department of Physics of Complex Systems, The Weizmann Institute of Science, Rehovot, Israel
}

(Received 5 November 1996)

\begin{abstract}
We report an experimental measurement of the temporal dependence of the area $A_{u s}$ in a twodimensional soap froth which has not been swept by the passage of soap films up to time $t$, as the froth coarsens from an initial time $t_{0}$ within the scaling regime. We find $A_{u s}$ scales with the mean cell area $\langle A\rangle$ as $A_{u s} \propto\langle A\rangle^{-\theta^{\prime}}$, with a first-passage exponent $\theta^{\prime}=1.16 \pm 0.02$; and for the average perimeter $\langle P\rangle:\langle P\rangle \propto\langle A\rangle^{\psi^{\prime}}$ and $A_{u s} \propto\langle P\rangle^{\phi^{\prime}}$ with values of the scaling exponents $\psi^{\prime}=0.49 \pm 0.01$ and $\phi^{\prime}=2.38 \pm 0.02$. We also find a scaling function in $A_{u s}(t) / A_{u s}\left(t_{0}\right)$ versus $\langle A(t)\rangle /\left\langle A\left(t_{0}\right)\right\rangle . \quad[\mathrm{S} 0031-9007(97) 02491-5]$
\end{abstract}

PACS numbers: 82.70.Rr, 02.50.-r, 05.70.Ln

The study of domain coarsening following a quench from a high-temperature disordered phase to a low temperature ordered one is relevant in many areas of physics ranging from the evolution of crystalline domains in metallurgy, to the initial stages of evolution of the Universe in cosmology [1]. Typically, domain patterns evolve into a scaling regime in which statistical pattern properties are time invariant and the average domain size increases as a power law $t^{\alpha}$. While these features have been well established experimentally and theoretically in classic statistical mechanical problems such as spinodal decomposition and Lifshitz-Slyozov processes, attention has been shifted more recently to properties related to the zerotemperature relaxational dynamics of certain theoretical models, which being unrelated to the usual response functions, are characterized by new nontrivial universal exponents [2-4]. These properties are related to the survival probability of domains, namely, how long until they are overrun by domain walls. The exponents have been calculated in various ways in the case of one-dimensional $q$-state Potts models [5-7], and estimated numerically from computer simulations of the two-dimensional versions of these models [8]. An approximate method for computing these exponents for arbitrary dimensions (for the Ohta-JasnowKawasaki model [9]) was given in [10,11].

To our knowledge, only one experimental work has addressed the measurement of an exponent of this type in a physical system [12]. The system consists of patterns of droplets condensing on a surface called breath figures, whose evolution is driven by droplet condensation and coalescence. Breath figures cannot be adequately modeled by domain coarsening of Potts models, and therefore a comparison between existing theoretical predictions based on these models and the experimental results was precluded.

In this paper, we present the results of an experimental measurement of exponents related to the zero-temperature Potts models. Our experiment was performed on a two-dimensional soap froth, a system whose evolution is well mimicked by $q$-state Potts model for large $q$
$[13,14]$. Coarsening two-dimensional soap froths have been studied extensively in recent years $[15,16]$. The various distributions characterizing the scaling state for the froths have been measured, and it has been established that the average bubble area increases linearly with time $(\alpha=1)[17,18]$. Theoretical results reproduce well this behavior $[19,20]$. In the present case, we focus our attention on the time dependence of $A_{u s}(t)$, the area inside all the bubbles which has not been swept by films up to time $t$, after letting the froth coarsen from an initial time $t_{0}$ within the scaling regime. According to the computer simulations of Derrida, de Oliveira, and Stauffer [8], the ratio $A_{u s}(t) / A_{u s}\left(t_{0}\right)$ decays as a power law $t^{-\theta}$ with $\theta \approx 0.86$, a prediction that we specifically check. They also introduce two other exponents related to the average perimeter $\langle P\rangle:\langle P\rangle \propto t^{\psi}$, and $A_{u s}(t) \propto\langle P\rangle^{\phi}$. They get the values of the scaling exponents $\psi=0.46$ and $\phi=2.0$. We have measured these values for soap froth in two dimensions and find similar value for $\theta$ but slightly different values for the other exponents.

Our experiment was performed in a $26.7 \times 36.8 \mathrm{~cm}^{2}$ chamber consisting of two $1.6 \mathrm{~cm}$ thick rectangular Plexiglas plates separated by a $0.16 \mathrm{~cm}$ thick spacer. Around 20000 soap bubbles of different sizes were pumped into the chamber initially so as to create a random froth from the outset. The scaling regime set in after about $6 \mathrm{~h}$, when there were around 6000 bubbles in the froth. The scaling state was identified by monitoring the stationarity of the distribution of number of sides. We have chosen two starting times, $t_{1}=12 \mathrm{~h}$ (2000 bubbles) and $t_{2}=$ $36 \mathrm{~h}$ (800 bubbles), for the measurements of unswept area. The latest time for the experiment is $160 \mathrm{~h}$ with 200 bubbles. A high resolution charge-coupled device camera $(1037 \times 1344$ pixels $)$ was used to capture images of the froth every 10 min during its evolution. To obtain the temporal dependence of the area within bubbles which was not swept by any bubble boundary up to a given time $t$, we formed groups of seven to twentyfive consecutive snapshots with a one-snapshot overlap 
between groups. All the snapshots within a group were then superimposed automatically, and swept sites were mapped out by image processing. Since the ten minute interval between snapshots of the evolution cannot capture very fast $T 1$ processes except for the states before and after the transition, there was a need to correct locally superimposed images where such processes took place. Starting from an initial pattern at an arbitrary time $t_{0}$ within the scaling regime, all the groups up to time $t$ were then superimposed. To illustrate the technique, we show in Fig. 1 a series of such superimposed images for different times. The white disconnected domains represent the areas unswept by soap films, while the shaded connected region represents the past and present positions of bubble boundaries.

In Fig. 2 we show plots of $A_{u s}(\tau)$ as a function of $\tau=t-t_{0}$, starting from two different values of $t_{0}=t_{1}$, and $t_{2}$, within the scaling regime of the same run. Powerlaw behavior sets in only a long time after $t_{0}$, during which the average bubble area changes by a factor of about 10. This limits the period over which power-law behavior is observed: only a decade for our earlier choice of $t_{0}$, while it is shorter for the later choice of $t_{0}$ (the small number of bubbles in the froth at the latest stage of evolution precludes continuation of the run for lack of statistics). A power-law fit to the late portion of the long data set (with $t_{0}=t_{1}$ ) yields $\theta=0.88 \pm 0.02$. Similar fit to the short data set (with $t_{0}=t_{2}$ ) yields smaller $\theta$ with large error bars. As for the other exponents, we get $\psi=0.35 \pm 0.02, \phi=2.38 \pm 0.02$. These results are consistent, despite their differences, with those obtained in the simulation of Derrida, de Oliveira, and Stauffer [8]. The discrepancy may be caused by the sensitive dependence on the absolute time origin $t^{\star}$. Without any a priori knowledge of the time origin, a better way is to use $\left(t-t^{\star}\right)$ as the dependent variable.

In order to avoid any bias of the exponents on the choice of time origin, we decide to use the mean bubble (a)

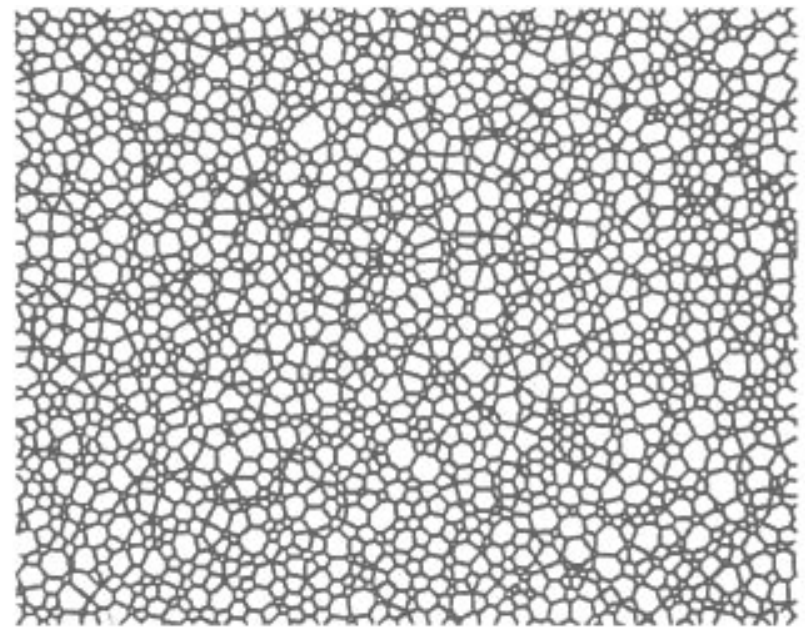

(b)

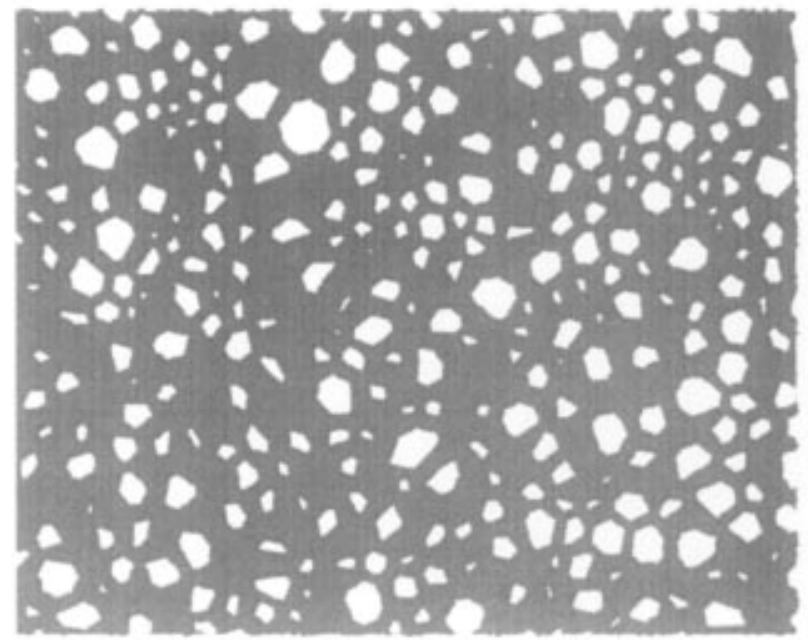

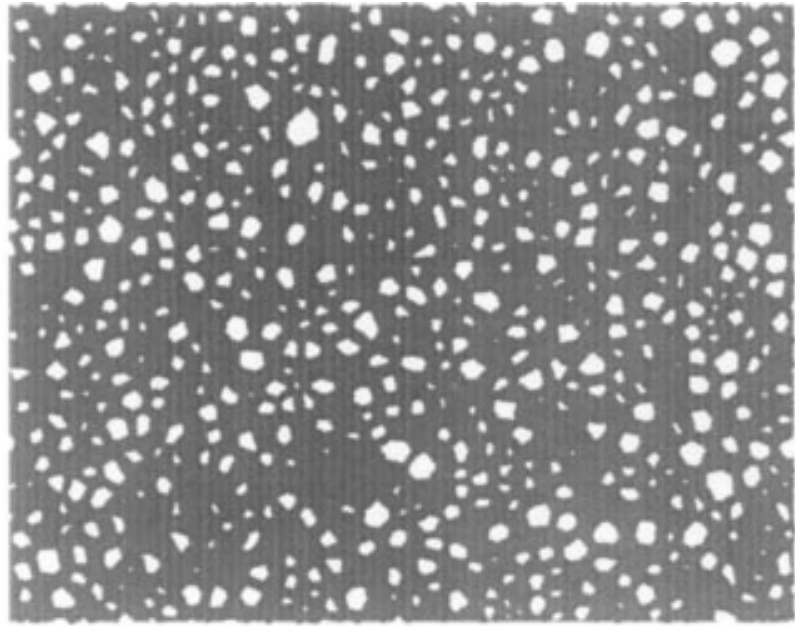

(c)

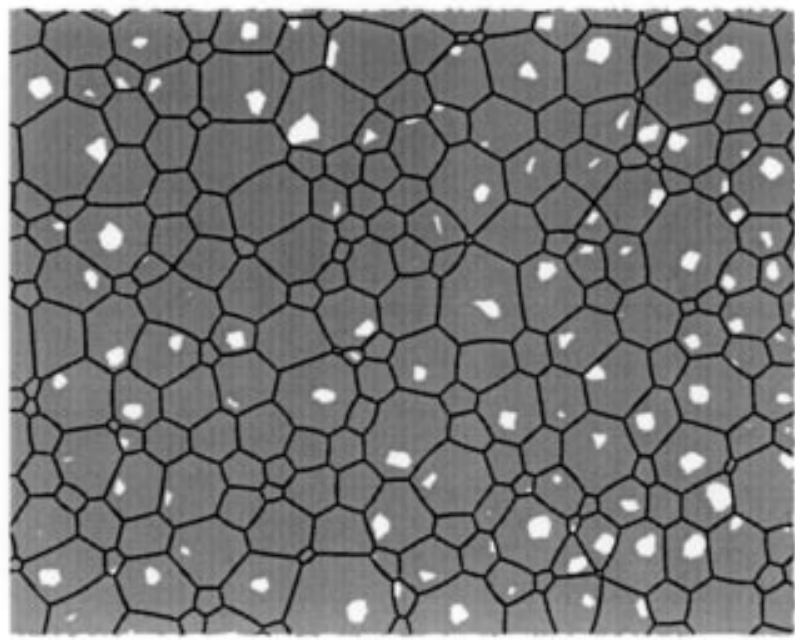

FIG. 1. Superimposed snapshots of a coarsening froth up to time $t$, starting from the initial state shown in (a), and after (b) $25.66 \mathrm{~h}$, (c) $75.00 \mathrm{~h}$, and (d) $150.00 \mathrm{~h}$. The white disconnected regions are unswept areas while the shaded region is the swept area. (d) The black connected lines are the boundaries of the froth at the latest time: they mark the survivors at the latest time. 


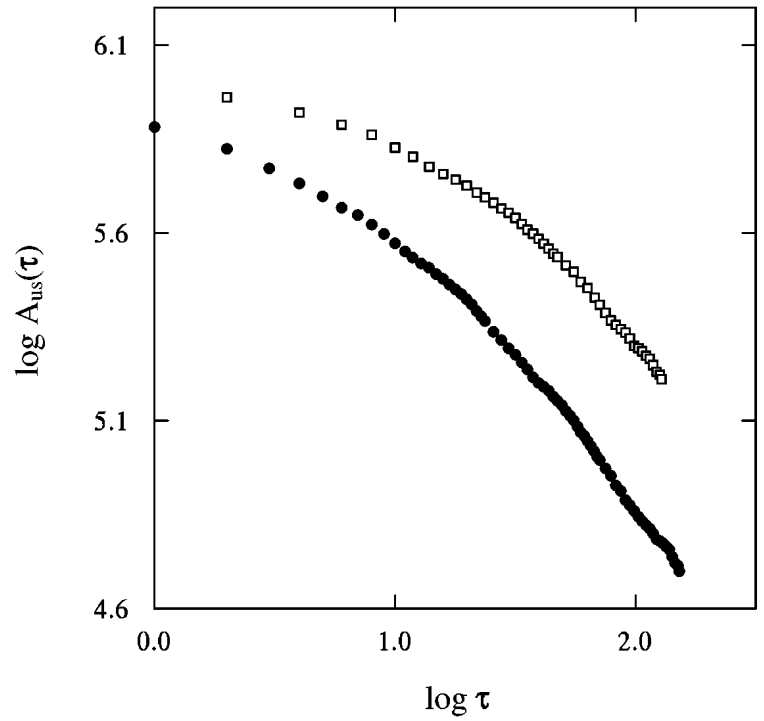

FIG. 2. Log-log plot of area of unvisited sites $A_{u s}(\tau)$ vs $\tau=t-t_{0}$ for $t_{0}=36.00 \mathrm{~h}$ (square) and $t_{0}=12 \mathrm{~h}$ (dot).

area $\langle A\rangle$ for our time scale. Indeed, the error introduced by the dependence of the exponent on $t_{0}$ is resolved by using $\langle A(t)\rangle$, since this is the physical quantity to be compared with the unswept area as the froth evolves. Furthermore, the errors in $A_{u s}$ and $\langle A\rangle$ compensate when we plot them against each other, but compound when we plot $A_{u s}$ vs time. This is obvious in the scaling regime as $\langle A(t)\rangle$ is linear in time. The choice of the origin of time is now resolved by noting that one can associate the origin with zero mean bubble area. In using $\langle A\rangle$ as the independent variable, we have the following definitions of scaling exponents: $A_{u s} \propto\langle A\rangle^{-\theta^{\prime}},\langle P\rangle \propto\langle A\rangle^{\psi^{\prime}}$, and $A_{u s} \propto$ $\langle P\rangle^{\phi^{\prime}}$ (note that $\phi^{\prime}=\phi$ ). Figure 3(a) shows the log-log plot of unvisited sites $A_{u s}(t)$ vs the average area $\langle A(t)\rangle$ for two initial times $t_{0}=t_{1}$ and $t_{0}=t_{2}$ as in Fig. 2. We see that Fig. 3(a) has larger ranges for the power-law fits supporting the use of $\langle A\rangle$ as a better choice of independent variable. Power-law fits to both sets of data give $\theta^{\prime}=$ $1.16 \pm 0.02$ and $\theta^{\prime}=1.30 \pm 0.05$ for the long and short data sets, respectively. Figure 3(b) shows a log-log plot of $A_{u s}(t)$ vs the $\langle P\rangle$, and the slope yields $\phi=\phi^{\prime}=2.38 \pm$ 0.02 using the long data set. Furthermore, a log-log plot of $\langle P\rangle$ vs $\langle A\rangle$ yields $\psi^{\prime}=0.49 \pm 0.02$. The values of $\theta^{\prime}, \psi^{\prime}$, and $\phi^{\prime}$ are consistent with the relation $\phi^{\prime}=\theta^{\prime} / \psi^{\prime}$.

Since the intrinsic scale of the system is $\langle A\rangle$, the time scales for all the physics are related to this quantity: the larger the $\langle A\rangle$, the slower the dynamics. Thus, the unswept area of a froth with initial mean area $\left\langle A\left(t_{1}\right)\right\rangle$ and the unswept area of the froth with initial mean area $\left\langle A\left(t_{2}\right)\right\rangle$ at a different time are the same when rescaled by their values at $t_{1}$ and $t_{2}$, respectively. Hence, we expect a scaling law for the unswept area in the following form $A_{u s}(t) / A_{u s}\left(t_{0}\right)=F\left(\langle A(t)\rangle /\left\langle A\left(t_{0}\right)\right\rangle\right)$. This is shown in Fig. 4 for two different $t_{0}=t_{1}, t_{2}$. The
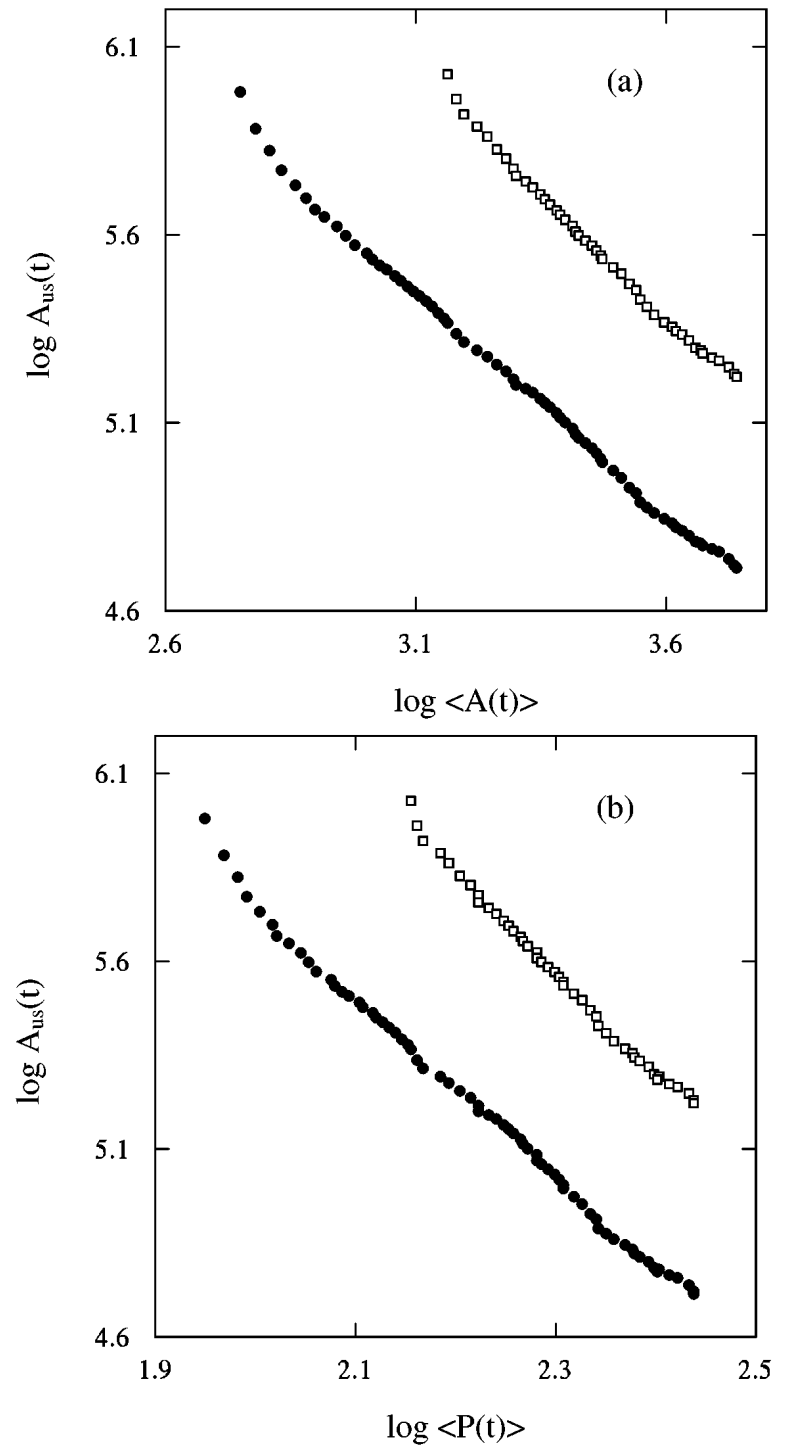

FIG. 3. (a) Log-log plot of area of unvisited sites $A_{u s}(t)$ vs $\langle A(t)\rangle$ for $t_{0}=36.00 \mathrm{~h}$ (square) and $t_{0}=12 \mathrm{~h}$ (dot). (b) Log$\log$ plot of area of unvisited sites $A_{u s}(t)$ vs $P$ for $t_{0}=36.00 \mathrm{~h}$ (square) and $t_{0}=12 \mathrm{~h}$ (dot).

collapse of the two sets of data supports the scaling function for $A_{u s}(t) / A_{u s}\left(t_{0}\right)$. While our data seem to have converged fairly well, we cannot rule out that the value of the measured exponents $\theta^{\prime}, \psi^{\prime}$, and $\phi^{\prime}$ would change slightly in a longer run on a larger system, and approach more closely the values predicted by the Potts models. However, if this discrepancy is real, it implies that the dynamics of the $q$-state Potts model do not describe all the aspects of froth coarsening.

In an effort to understand why $A_{u s}(t)$ decays with a nontrivial exponent $\theta^{\prime}$, we find it instructive to compare the pattern of unswept domains at the latest time measured with the surviving froth at this same time. The superposition of these two is shown in Fig. 1(d). Notice that while a large part of unswept domains are located 


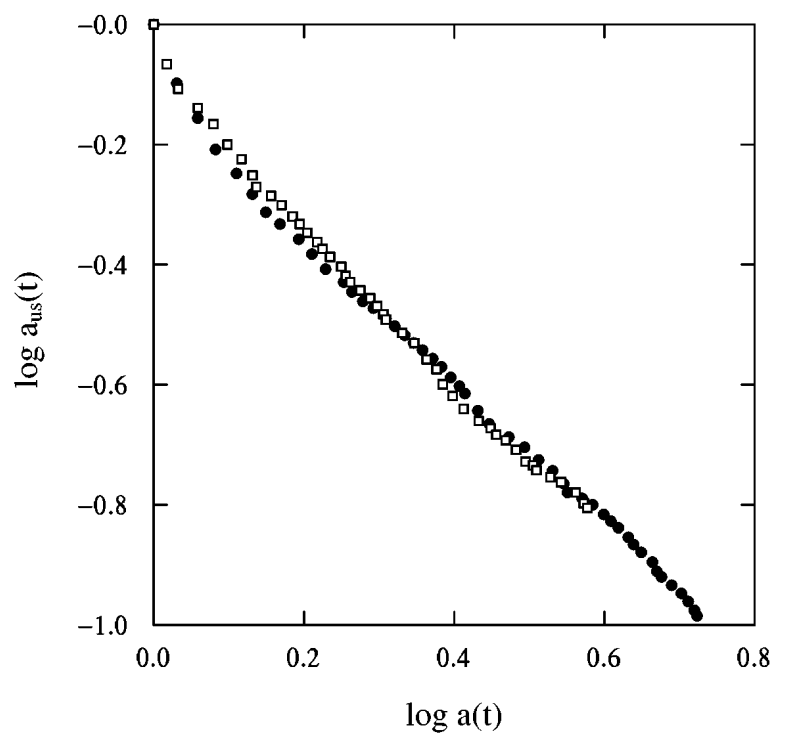

FIG. 4. Log-log plot of the ratio $a_{u s}(t)=A_{u s}(t) / A_{u s}\left(t_{0}\right)$ vs $a(t)=\langle A(t)\rangle /\left\langle A\left(t_{0}\right)\right\rangle$. There are two values of $A\left(t_{0}\right)$ for $t_{0}=$ $36.00 \mathrm{~h}$ (square) and $t_{0}=12 \mathrm{~h}(\mathrm{dot})$.

well within the interior of the surviving bubbles, only a small number of these domains touch the bubble boundaries. The changes of the unswept area $A_{u s}(t)$ at $t$, or a short time thereafter, $t+\delta t$, come mainly from these domains, while the domains in the bubble interiors only contribute to the change of $A_{u s}(t)$ at a later time due to the slow diffusion process. However, some of the domains, even when they are located well inside surviving bubbles, can be swept by the large boundary movements induced by the very fast relaxations of $T 1$ and $T 2$ processes as observed in the experiment for the whole duration of the coarsening of the froth. While it is difficult to estimate the effect of these processes which are absent in the Potts models, it is possible that they are partly responsible for the discrepancy between the measured $\theta^{\prime}=1.16$ and the value $\theta^{\prime}=1$ as predicted by the $q$-state Potts models [21] and recent simulations for the surviving bubbles [22].

Thus far, we have discussed only the case where the starting times $t_{0}$ are in the scaling regime, where $\langle A(t)\rangle \propto$ $t$. However, it will be of interest for the case where $t_{0}$ is chosen outside the scaling regime. If all the dynamics of soap froth evolution can be described by the evolution of average bubble area, and that this is the only physical time scale relevant to the problem of unvisited sites, then the measured exponents should be the same even if $t_{0}$ is chosen outside of the scaling regime.

We acknowledge useful correspondence with B. Derrida, B. Levitan, and E. Domany. W. Y. T. acknowledges support from the Direct Allocation Grant No. 96/97.SC22 of the Hong Kong University of Science and Technology. K. Y.S. acknowledges support from the Hong Kong Telecom Institute of Information Technology.

[1] For a review see A. J. Bray, Adv. Phys. 43, 357 (1994).

[2] H. Furukawa, Phys. Rev. B 40, 2341 (1989).

[3] D. S. Fisher and D. A. Huse, Phys. Rev. B 38, 373 (1988).

[4] T. J. Newman, A. J. Bray, and M. A. Moore, Phys. Rev. B 42, 4514 (1990).

[5] B. Derrida, A. Bray, and C. Godreche, J. Phys. A 27, L357 (1994).

[6] J. Cardy, J. Phys. A 28, L19 (1995).

[7] B. Derrida, V. Hakim, and V. Pasquier, Phys. Rev. Lett. 75, 751 (1995).

[8] B. Derrida, P. M. C. de Oliveira, and D. Stauffer, Physica (Amsterdam) 224A, 604 (1996).

[9] T. Ohta, D. Jasnow, and K. Kawasaki, Phys. Rev. Lett. 49, 1223 (1982).

[10] B. Derrida, V. Hakim, and R. Zeitak, Phys. Rev. Lett. 77, 2871 (1996).

[11] S. N. Majumdar, C. Sire, A. J. Bray, and S. Cornell, Phys. Rev. Lett. 77, 2867 (1996).

[12] M. Marcos-Martin, D. Beysens, J. P. Bochaud, C. Godreche, and I. Yekutieli, Physica (Amsterdam) 214A, 396 (1995).

[13] J. A. Glazier, M.P. Anderson, and G. S. Grest, Phys. Mag. B 62, 6115 (1990).

[14] E. A. Holm, J. A. Glazier, D. J. Srolovitz, and G. S. Grest, Phys. Rev. A 43, 2662 (1991).

[15] J. Stavans, Rep. Prog. Phys. 56, 733 (1993).

[16] D. Weaire and N. Rivier, Contemp. Phys. 25, 59 (1984).

[17] J. A. Glazier, S. Gross, and J. Stavans, Phys. Rev. A 36, 306 (1987).

[18] W. Y. Tam and K. Y. Szeto, Phys. Rev. E 53, 877 (1996).

[19] H. Flyvbjerg, Phys. Rev. E 47, 4037 (1993); Physica (Amsterdam) 194A, 298 (1993).

[20] J. Stavans, E. Domany, and D. Mukamel, Europhys. Lett. 15, 479 (1991).

[21] C. Sire and S. N. Majumdar, Phys. Rev. E 52, 244 (1995).

[22] B. Levitan, E. Slepyan, O. Krichevsky, J. Stavans, and E. Domany, Phys. Rev. Lett. 73, 756 (1994); B. Levitan and E. Domany, Phys. Rev. E 54, 2766 (1996). 